\title{
Growing tissue grafts on humanoid robots: a future strategy in regenerative medicine?*
}

\author{
Pierre-Alexis Mouthuy, Andrew Carr \\ NIHR Oxford Musculoskeletal Biomedical Research Unit, Biomedical Research \\ Centre, Nuffield Department of Orthopaedics, Rheumatology and Musculoskeletal \\ Sciences, University of Oxford, Oxford OX3 7LD, UK \\ Email: pierre-alexis.mouthuy@ndorms.ox.ac.uk
}

\begin{abstract}
* This manuscript has been accepted for publication in Science Robotics. This version has not undergone final editing. Please refer to the complete version of record at robotics.sciencemag.org/content/2/4/eaam5666. The manuscript may not be reproduced or used in any manner that does not fall within the fair use provisions of the Copyright Act without the prior, written permission of AAAS.
\end{abstract}

Over the last decade exciting progress has been made in the development of humanoid robots. The significant potential future value of humanoids includes applications ranging from personal assistance to medicine and space exploration. In particular, musculoskeletal humanoids (such as Kenshiro and Eccerobot) were developed to interact with humans in a safer and more natural way $(1,2)$. They aim to closely replicate the detailed anatomy of the human musculoskeletal system including muscles, tendons and bones.

With their structures activated by artificial muscles, musculoskeletal humanoids have the ability to mimic more accurately the multiple degrees of freedom and the normal range of forces observed in human joints. As a result, it is not surprising that they offer new opportunities in science and medicine. Here, we suggest that musculoskeletal robots may assist in the growth of musculoskeletal tissue grafts for tissue transplant applications.

In ageing populations, musculoskeletal tissue disorders and injuries are a growing health, social and economic burden. Pain and lack of mobility are common problems due to failure of tissues such as tendon, ligament, bone and cartilage. A promising repair strategy is to engineer tissue grafts. Tissue engineering is enabled by the development of bioreactor systems, which control the environmental conditions necessary for maintaining living cells and tissues outside the body. They also provide chemical and mechanical stimulations that promote the differentiation of particular cell phenotypes within the tissue construct (3). However, in order to create functional tissue grafts, more advanced bioreactors are needed. In particular, current bioreactors provide stimuli that fail to mimic the real mechanical environment for cells and this hinders or prevents the fabrication of clinically relevant grafts.

Mechanical stimulation is central to the successful development of musculoskeletal tissues both in vivo and in vitro. Compared to static conditions, dynamic stresses applied in vitro generally improve the mechanical properties of engineered constructs, and lead to larger cell numbers, greater matrix deposition and better cell differentiation (4-6). Moreover, multi-dimensional strains are known to improve the functionality of engineered tissues (7). Overall, the evidence in the literature suggests that mechanical stimulation in vitro should mimic as closely as possible stresses experienced by the tissues in vivo. 
However, bioreactors systems developed so far in musculoskeletal tissue engineering are relatively rudimentary. Typical tissue bioreactors consist of a hard casing used as a culture chamber (in which tissue constructs are resident) coupled with a linear actuator, which subjects constructs to cyclic loading. These desktop bioreactors are usually able to apply uniaxial or biaxial stresses with a programmable loading regime.

The main limitation of current bioreactors is that they poorly mimic the mechanical loadings that are experienced in the body. To produce clinically relevant grafts, future bioreactors will need to:

- provide multidirectional stresses by a combination of tension, torsion, compression and shear stresses

- adapt the loading regime to each individual tissue in consideration of their anatomical location

- enable the fabrication of tissue constructs with dimensions similar to their native counterparts.

Bearing the considerations above in mind, it makes sense to create advanced bioreactors with structures, dimensions and mechanics similar to those of the human body. In this context, humanoid musculoskeletal robots become highly relevant. By mimicking the human skeletal architecture and the body movements in different activities, they could help to overcome the limitations of current bioreactors.

Designing "humanoid-bioreactor" systems would involve several considerations, such as:

- How would the robot interact with its environment? The ability of humanoids to freely interact with their environment and real objects could be an advantage compared to desktop bioreactors. This may provide more realistic stresses to tissue constructs and eventually achieve grafts with better functionality or with tailored properties.

- How would the humanoid-bioreactor be actuated? In robots such as Kenshiro and Eccerobot, the main muscles and tendons are mimicked with rubber-like materials activated by inelastic strings, themselves attached to spindles driven by motors. However, more recent robots have also used pneumatic artificial muscles, suggesting that other materials developed in soft robotics (8) such as electroactive polymer artificial muscles could also be implemented in musculoskeletal humanoids to provide better mechanics. It is worth mentioning that, although replacing the actuation system by engineered tissues is not envisioned here, combining humanoid with cell-based actuators (9) may also become a strategy in the future.

- How would forces be sensed and controlled? It is important to ensure proper tissue development. In particular, monitoring the variations of the construct's mechanical properties as the tissue grows and matures may help humanoids to optimise the loading regime. For such applications, flexible and stretchable sensors such as those used in soft robotics may be appropriate. Typical sensors used in current bioreactors might also help humanoids to assess (and respond to) the advancement of tissue formation, for example by monitoring the $\mathrm{pH}$ and the glucose and lactate concentrations in the culture medium.

Humanoid-bioreactor systems may open up numerous opportunities in medicine, science and technology, in the spirit of "science for robotics and robotics for science" (10). In particular, they may: 
- lead towards the fabrication of more clinically relevant musculoskeletal tissue grafts and, in particular, allow for personalized tissue graft development by matching the robot's morphology and mechanics to the patient's needs.

- support the development of multiphasic constructs, such as bone-tendonmuscle grafts, which is an important area of development in tissue engineering because failure during healing often occurs at the interface between tissues.

- provide further insights into mechanobiology and tissue healing mechanisms.

- enable faster screening of potential scaffolds for musculoskeletal repair and reduce the use of animal models in pre-clinical trials.

- accelerate the development of safer musculoskeletal robots with more natural movements and lead to the development of bio-hybrid humanoids, considering the emergence of cell-based actuators.

In summary it is now both technically possible and scientifically pertinent to explore in greater detail the potential of humanoids as tools for regenerative medicine. Advances in this field could lead to exciting applications across multiple disciplines.

\section{References}

1. M. Inaba, Musculoskeletal Humanoid as Intelligent Robot Frontier. Journal of the Robotics Society of Japan 28, 666-671 (2010).

2. A. Diamond, R. Knight, D. Devereux, O. Holland, Anthropomimetic Robots: Concept, Construction and Modelling. Int J Adv Robot Syst 9, 209 (2012).

3. I. Martin, D. Wendt, M. Heberer, The role of bioreactors in tissue engineering. Trends in Biotechnology 22, 80-86 (2004).

4. K. T. S. Palomares et al., Mechanical stimulation alters tissue differentiation and molecular expression during bone healing. Journal of orthopaedic research : official publication of the Orthopaedic Research Society 27, 11231132 (2009).

5. C. A. Powell, B. L. Smiley, J. Mills, H. H. Vandenburgh, Mechanical stimulation improves tissue-engineered human skeletal muscle. American journal of physiology. Cell physiology 283, C1557-1565 (2002).

6. D. W. Youngstrom, I. Rajpar, D. L. Kaplan, J. G. Barrett, A bioreactor system for in vitro tendon differentiation and tendon tissue engineering. Journal of orthopaedic research : official publication of the Orthopaedic Research Society 33, 911-918 (2015).

7. G. H. Altman et al., Advanced bioreactor with controlled application of multidimensional strain for tissue engineering. Journal of biomechanical engineering 124, 742-749 (2002).

8. C. Laschi, B. Mazzolai, M. Cianchetti, Soft robotics: Technologies and systems pushing the boundaries of robot abilities. Science Robotics $\mathbf{1}$, (2016).

9. L. Ricotti, A. Menciassi, Bio-hybrid muscle cell-based actuators. Biomedical microdevices 14, 987-998 (2012).

10. G.-Z. Yang et al., Science for robotics and robotics for science. Science Robotics 1, (2016). 